\title{
POBREZA CERO: LA METODOLOGÍA DE LAS PROMESAS ROTAS
}

POVERTY ZERO: THE METHODOLOGY OF THE BROKEN PROMISES

\author{
GUIDO ZACK \\ FEDERICO FAVATA \\ FLORENCIA FARES
}

Escuela de Economía y Negocios

Universidad Nacional de San Martín

ARGENTINA 


\title{
RESUMEN
}

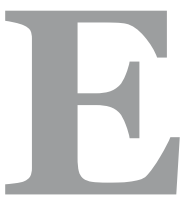

n 2016 el INDEC volvió a difundir los datos de pobreza e indigencia de la Argentina, luego de haberlos interrumpido en 2013 por resultar estos muy alejados de la realidad. El instituto de estadística implementó también una actualización metodológica que hace a las nuevas series no comparables en relación a las anteriores. En este artículo se detallan todas las modificaciones realizadas y se muestra su impacto marginal y total en las tasas y en las brechas de pobreza e indigencia. Asimismo, a partir de los datos del segundo trimestre de 2016, se evalúan las combinaciones de crecimiento y mejora en la distribución del ingreso necesarias para la eliminación de la pobreza y/o la indigencia para finales de 2019 y 2023.

Palabras clave: pobreza, indigencia, metodología, Argentina.

\begin{abstract}
In 2016 INDEC started publishing again poverty and extreme poverty statistics, after having interrupted them in 2013 because they were very far from reality. The statistic institute also updated the methodology used, which makes the new series not comparable with previous ones. This article details all the modification made and shows its marginal and total impact on rates and gaps of poverty and extreme poverty. Additionally, using second quarter of 2016 data, all combinations of growth and income distribution improvement to achieve the objective of total elimination of poverty and / or extreme poverty by the end of 2019 and 2023 are evaluated.
\end{abstract}

Keywords: poverty, extreme poverty, methodology, Argentina.

\section{INTRODUCCIÓN}

En los últimos años, el debate acerca de la pobreza en la Argentina ha girado más en torno a cuál es su nivel real que a las formas de reducirla. Eso se debió a que la sistemática subestimación de las cifras de inflación entre 2007 y 2015 por parte del Instituto Nacional de Estadísticas y Censos de la República Argentina (INDEC) se trasladó a la canasta básica total (CBT) y alimentaria (CBA). Como no podía ser de otra manera, la subestimación de las 
líneas de pobreza e indigencia impactaron de lleno en los indicadores sociales mostrando valores mucho menores a los reales. En efecto, según el INDEC, la pobreza durante el primer semestre de 2013 alcanzó solo al 4,7\% de la población y la indigencia al 1,4\%, cuando según diversas estimaciones privadas estos porcentajes eran hasta seis veces superiores en el primer caso y cuatro en el segundo.

Como parte del intento del nuevo gobierno por devolver la credibilidad al organismo estadístico, a finales de septiembre último se volvieron a publicar los datos oficiales de pobreza e indigencia, los cuales - para sorpresa de muchos - se situaron muy por encima de la mayor parte de los cálculos privados. Así, mientras que la mayor parte de las consultoras estimaban la pobreza de 2015 en torno al 20\% de la población y la indigencia alrededor del $5 \%$, el INDEC (2016a) informó que durante el segundo trimestre de 2016 estas tasas se ubicaron en 32,2\% y $6,3 \%$, respectivamente. ¿ंPuede la pobreza haber aumentado casi un $50 \%$ en solo un año? ¿Por qué la pobreza aumentó tanto más que la indigencia?

La respuesta a estas preguntas es, simplemente, que los datos de condiciones sociales que difundió este año el INDEC no son comparables con los datos previos ni con las estimaciones de las consultoras privadas. El motivo es que se realizó una actualización metodológica de varios aspectos, entre los que se encuentran una mejor especificación de las necesidades energéticas en términos de adulto equivalente; la utilización de una CBA y CBT específica para cada región (en lugar de usar la canasta del Gran Buenos Aires para todo el país); y una actualización en la estructura de consumo a partir del reemplazo de la Encuesta Nacional de Gastos de los Hogares (ENGHo) de 1985/6 por la de 2004/5. Esta última modificación, la que más diferencias genera en los indicadores sociales, implica dos cosas. La primera, la inclusión de algunos bienes en la CBA y el cambio en la contribución de muchos de los productos preexistentes en el total de la canasta. La segunda, un aumento en la inversa del Coeficiente de Engel (ICE), es decir, en el factor que se utiliza para expandir el valor de la línea de indigencia para calcular la línea de pobreza.

De esta manera, el presente artículo tiene como objetivo principal detallar todas las actualizaciones metodológicas realizadas por el INDEC en su última medición de pobreza e indigencia, lo que se realiza en la siguiente sección. Luego, se estiman las series de tasa y brecha de pobreza e indigencia con la metodología vieja y con cada una de las actualizaciones metodológicas por separado, de forma de poder observar cuáles generan cambios más significativos sobre los indicadores. En la cuarta sección se calculan las curvas de isopobreza e isoindigencia a partir de los datos resultantes de la metodología vieja y nueva, de modo de identificar cuán cerca o lejos está el gobierno actual de poder cumplir con su promesa de campaña de pobreza cero para el fin de actual mandato presidencial en 2019. Por último, se esbozan algunas reflexiones finales. 


\section{LA METODOLOGÍA DEL CÁLCULO DE LA POBREZA E INDIGENCIA POR INGRESOS}

Los indicadores de pobreza e indigencia calculados por el INDEC se basan en el método indirecto o de "línea". Este método, primeramente, determina el requerimiento energético y las recomendaciones de nutrientes de cada individuo según el sexo y la edad. Se establece una unidad de referencia, el adulto equivalente, el cual está compuesto por las personas de un sexo y rango de edad determinado, para luego medir al resto de la población de referencia en relación a ese adulto equivalente. Así, hasta la actualización metodológica del año pasado, era considerado adulto equivalente a todo individuo de sexo masculino entre 30 y 59 años, el cual necesita 2700 kilocalorías (Kcal) por mes para su correcta nutrición y desarrollo. De esta forma, una mujer de entre 18 y 59 años era considerada 0,74 adultos equivalentes, dado que necesita $2000 \mathrm{Kcal}$, el $74 \%$ de las 2700 Kcal del adulto equivalente.

Aquí se encuentra el primer cambio metodológico que aplicó el INDEC en 2016, aunque su efecto sobre los indicadores sociales es muy marginal. El adulto equivalente pasó a ser todo individuo hombre de entre 30 y 60 años, y se aumentó su requerimiento energético a 2750 Kcal. Luego, también se ajustaron los requerimientos energéticos del resto de la población y se procedió a una mayor especificación. Así, por ejemplo, se desagregó al grupo de mujeres de entre 18 y 59 años en tres: las mujeres de entre 18 y 29 años necesitan 2106 Kcal (o,766 adultos equivalentes), entre 30 y 45 años necesitan $2111 \mathrm{Kcal}(0,768$ adultos equivalentes) y entre 46 y 60 años necesitan 2090 Kcal (o,76 adultos equivalentes; ver tabla A.2 en el anexo).

Una vez establecidos los requerimientos energéticos de todos los grupos poblaciones por sexo y edad, se traducen las kilocalorías necesarias en bienes alimentarios, según el patrón de consumo de la población de referencia que se desprende de la ENGHo. Así surge la CBA, la cual es valuada a precios de mercado a partir de los datos del Índice de Precios al Consumidor (IPC), obteniéndose como resultado la línea de indi-gencia. De esta manera, los hogares cuyos ingresos familiares por adulto equivalente (IFAE) no superen esa línea son considerados indigentes, debido a que sus ingresos no alcanzan para proveer a la familia de las kilocalorías necesarias para su correcta nutrición y desarrollo. En cambio, los hogares cuyos IFAE sean mayores a la CBA no son considerados indigentes.

El segundo cambio en la medición del indicador, de mayor importancia que el mencionado anteriormente, fue la actualización de los productos que componen la CBA, así como de sus representaciones dentro del total. Esto se dio dado que se actualizó el patrón de consumo resultante de la ENGHo 1985/6 para pasar a basarse en la ENGHo 2004/5. La diferencia más destacada entre la CBA anterior y la actual fue la incorporación de nuevos productos, como por ejemplo bebidas alcohólicas, pescado, yogur, manteca, y fiambres. También se registraron cambios en las cantidades de los bienes. Por ejemplo el pan aumentó en 690 gramos (gr.), el 
arroz 570 gr., los fideos 450 gr., las hortalizas 1800 gr., las frutas 930 gr. y la leche 1320 gr., entre otros. Por su parte, las galletitas dulces disminuyeron en 510 gr., la papa en 540 gr., la batata 180 gr., el azúcar 210 gr., la sal gruesa 90 gr., entre otros (ver tabla A.1 en el anexo).

Obtenida la CBA y la línea de indigencia, el siguiente paso es calcular la CBT o línea de pobreza, es decir el IFAE a partir del cual los miembros de una familia dejan de ser considerados pobres. La CBT incluye, además de los productos de la CBA, otros no alimentarios básicos, como ser vestimenta, transporte, educación, salud, etc. Esta se calcula expandiendo la CBA por un coeficiente resultante nuevamente de la ENGHo. Se define la población de referencia como aquella cuyo consumo de alimentos satisface las necesidades mínimas alimentarias y se calcula su Coeficiente de Engel, es decir, el porcentaje del gasto total que representa el gasto en alimentos. A partir de este coeficiente (o, mejor dicho, de su inversa), se determina cuánto mayor debe ser la $\mathrm{CBT}$ en relación a la CBA. En términos prácticos, lo que se hace es multiplicar la CBA por el ICE para obtener la CBT.

$$
\begin{gathered}
\text { Coeficiente de Engel }=\frac{\text { Gasto alimentario }}{\text { Gasto total }} \\
C B T=C B A * I C E=C B A\left(\frac{\text { Gasto alimentario }}{\text { Gasto total }}\right)^{-1}=C B A \frac{\text { Gasto alimentario }}{\text { Gasto total }}
\end{gathered}
$$

Una vez determinado a través de la ENGHo el porcentaje del gasto total que representa el gasto en alimentos para la población de referencia, el Coeficiente de Engel se va actualizando a partir de la variación de precios de los bienes y servicios que componen la CBT, bajo el supuesto de que las cantidades consumidas no se modifican ante dichas variaciones de precios ni tampoco ante variaciones en el ingreso (elasticidad precio y precio cruzada de la demanda igual a cero, y elasticidad ingreso de la demanda idéntica para todos los bienes y servicios). Así, en caso de que los precios de los alimentos se incrementen en mayor (menor) proporción que los precios de los productos no alimentarios, la ICE va a mostrar una baja (suba).

He aquí la tercera, y posiblemente más significativa, actualización metodológica realizada por el INDEC en la medición de pobreza de 2016. Hasta la medición anterior, la ICE era estimada a partir de la ENGHo 1985/6 y actualizada a través de las variaciones de precios de los componentes de la CBT. A partir de 2016 se actualizó la estructura de gasto mediante la ENGHo 2004/5, ajustándola desde entonces por las variaciones de precios. Esto genera una importante diferencia en la nueva ICE utilizada. Para dar una idea, mientras que con la ENGHo 1985/ 6 la ICE de 2006 se ubicaba entre 2,15 y 2,20, con la ENGHo 2004/5 este coeficiente se eleva a alrededor de 2,65 (INDEC, 2016b). Si bien esta mayor ICE no tiene influencia sobre los indicadores de indigencia, sí los tiene sobre los de pobreza. En efecto, al aumentar el ICE, también se incrementa la línea de pobreza y, por lo tanto, ciertos hogares que antes tenían 
ingresos suficientes para adquirir una CBT por adulto equivalente, con la nueva ICE no los tienen. En resumen, la tasa de pobreza es mayor. Este es el principal motivo por el cual el salto en la tasa de pobreza a partir de la actualización metodológica es mucho más importante en comparación al aumento en la tasa de indigencia.

El último cambio metodológico que introdujo el INDEC fue el cálculo de una CBA y CBT específica para cada una de las regiones de la Argentina. Hasta ahora, todos los ingresos de las familias del país se comparaban con las CBA y CBT resultantes de los patrones de consumo del Gran Buenos Aires. Pero, a partir de los datos de la ENGHo 2004/5, se confeccionaron tantas CBA como regiones (Gran Buenos Aires, Cuyo, Noreste, Noroeste, Pampeana y Patagónica). Asimismo, se calculó una ICE para cada una de las regiones, según sus relaciones de gasto entre alimentos y total, por lo que también se cuenta con una CBT específica a sus patrones de consumo. Cada una de estas canastas se actualiza en el tiempo a partir de las variaciones de los precios de productos de cada una de las regiones (INDEC, 2004).

En definitiva, las actualizaciones metodológicas propuestas por el INDEC para la medición de los indicadores de indigencia y pobreza pueden resumirse en cuatro aspectos: los ajustes en los requerimientos kilo-calóricos de los individuos según sexo y edad, la actualización a partir de la ENGHo 2004/5 de los productos componentes de la CBA y su participación en el total del consumo alimentario, la actualización de la ICE también a partir de los datos resultantes de la ENGHo 2004/5y, finalmente, la construcción de una CBA y CBT específica por cada región de la Argentina.

En lo que sigue, entonces, se procede a calcular las tasas y brechas de pobreza e indigencia, tanto con la metodología vieja como con cada una de las novedades metodológicas por separado y con la metodología nueva completa. Así se podrá identificar a qué cambio metodológico se deben las principales diferencias. Cabe aclarar que durante el período 2007-2015, es decir, mientras las estadísticas de inflación publicadas por el INDEC no reflejaban correctamente la realidad, los precios de los productos de la CBA y CBT se ajustaron mediante índices privados. Asimismo, ante la imposibilidad de hacer una actualización de las canastas regionales por falta de datos, no se pudo calcular el efecto marginal de este cambio metodológico.

\section{EVOLUCIÓN DE LOS INDICADORES SOCIALES SEGÚN LA VIEJA Y LA NUEVA METODOLOGÍA}

Las tasas de pobreza e indigencia, posiblemente los indicadores sociales más conocidos, miden la incidencia del fenómeno, es decir, qué porcentaje de la población total tiene un IFAE menor a la CBT y CBA respectivamente. En otras palabras, todos los individuos pobres o indigentes poseen la misma ponderación, sin importar su nivel de ingreso. Lo único relevante es si es mayor o menor a la línea. Por su parte, los indicadores de brecha miden la profundidad del 
fenómeno, es decir, a qué distancia se encuentra cada individuo pobre o indigente de dejar de serlo. Por ejemplo, si la línea de pobreza es $\$ 1000$ y el individuo tiene un IFAE de $\$ 700$, entonces su brecha es igual a $\$ 300$ ( $\$ 1000-\$ 700)$. Sumando la brecha de todos los individuos pobres, se obtiene la brecha de pobreza total de la economía, en otras palabras, cuánto es el monto mínimo de dinero necesario para eliminar la pobreza. Por motivos de simplicidad expositiva, en este artículo las brechas se miden en términos del ingreso total. De esta forma, estos indicadores mostrarán cuál es el porcentaje mínimo del ingreso total de economía necesario para eliminar la pobreza y la indigencia.

$$
\begin{gathered}
\text { Tasa de pobreza o indigencia }=\frac{1}{N} \sum_{i=1}^{l}\left(\frac{z-y_{i}}{z}\right)^{o} \text { si } z>y_{i}=\frac{1}{N} \sum_{i=1}^{l} 1 \text { si } z>y_{i} \\
=\frac{\text { Cantidad de pobres } \text { o indigentes }}{\text { población total }(N)}
\end{gathered}
$$$$
\text { Brecha de pobreza o indigencia }=\frac{1}{N} \sum_{i=1}^{l}\left(z-y_{i}\right)^{1} \text { si } z>y^{i}
$$

$$
=\frac{\text { ingreso mínimo necesario para eliminar la pobreza o indigencia }}{\text { ingreso total }(Y)}
$$

Donde " $z$ " es la línea de pobreza o indigencia e "yi" es el IFAE del individuo "i”, "N" es la población total e "Y" es el ingreso total de la economía.

Nótese que la diferencia entre los indicadores se encuentra en el exponente de la ecuación. En el caso de las tasas, como el exponente es igual a cero, cada individuo cuyo IFAE es menor a la línea, toma valor a uno (cualquier número elevado a la cero es igual a uno). En cambio, en el caso de las brechas, como el exponente es igual a uno, cada individuo cuyo IFAE es menor a la línea, toma valor igual a la diferencia entre la línea y el IFAE. Así, cuanto menor sea el IFAE, mayor será la brecha, y los individuos toman una mayor ponderación.

Los datos del presente artículo se toman de la serie completa de la Encuesta Permanente de Hogares (EPH) en su modalidad continua, es decir, una serie trimestral que va desde el segundo trimestre de 2003 hasta el segundo de 2016 (aunque se presen-tan cuatro observaciones sin datos: el tercer trimestre de 2007, el tercero y cuarto de 2015, y el primero de 2016).

Como puede observarse en el gráfico 1, todas las tasas de pobreza muestran una evolución similar, pero se diferencian en el nivel. En todos los casos el periodo inicial muestra los valores más elevados, para reducirse rápidamente hasta 2007, luego seguir disminuyendo aunque a una velocidad menor, hasta 2012 cuando se estancan e incluso aumentan durante 2014 y 2016. Según la metodología vieja, la pobreza hacia finales de 2003 y principios de 2004 afectaba a entre el 45\% y 50\% de la población total argentina, mientras que al segundo trimestre de 2015 
este porcentaje había bajado al 20\%, aumentando al año siguiente 1 punto porcentual (p.p.). Como puede notarse también en el gráfico, el cambio metodológico de la especificación de las necesidades energéticas en términos de adulto equivalente generó una reducción en todos los valores, aunque de magnitud insignificante. Por su parte, la actualización de la CBA provocó un incremento en las tasas de entre 2,2 y 4,2 p.p., con un promedio de 3,5 p.p. El cambio en la ICE es el que indujo mayores diferencias con una suba de entre 5 y 8,4 p.p., promediando 6,8 p.p. en todo el período. En definitiva, el cambio metodológico completo generó un aumento en la tasa de pobreza promedio de 10,1 p.p., que va desde 7,8 p.p. y 12,6 p.p. Justamente, este último valor es la diferencia existente entre la metodología vieja y la nueva en el segundo semestre de 2016.

Gráfico 1: Tasa de pobreza (\% de la población total)

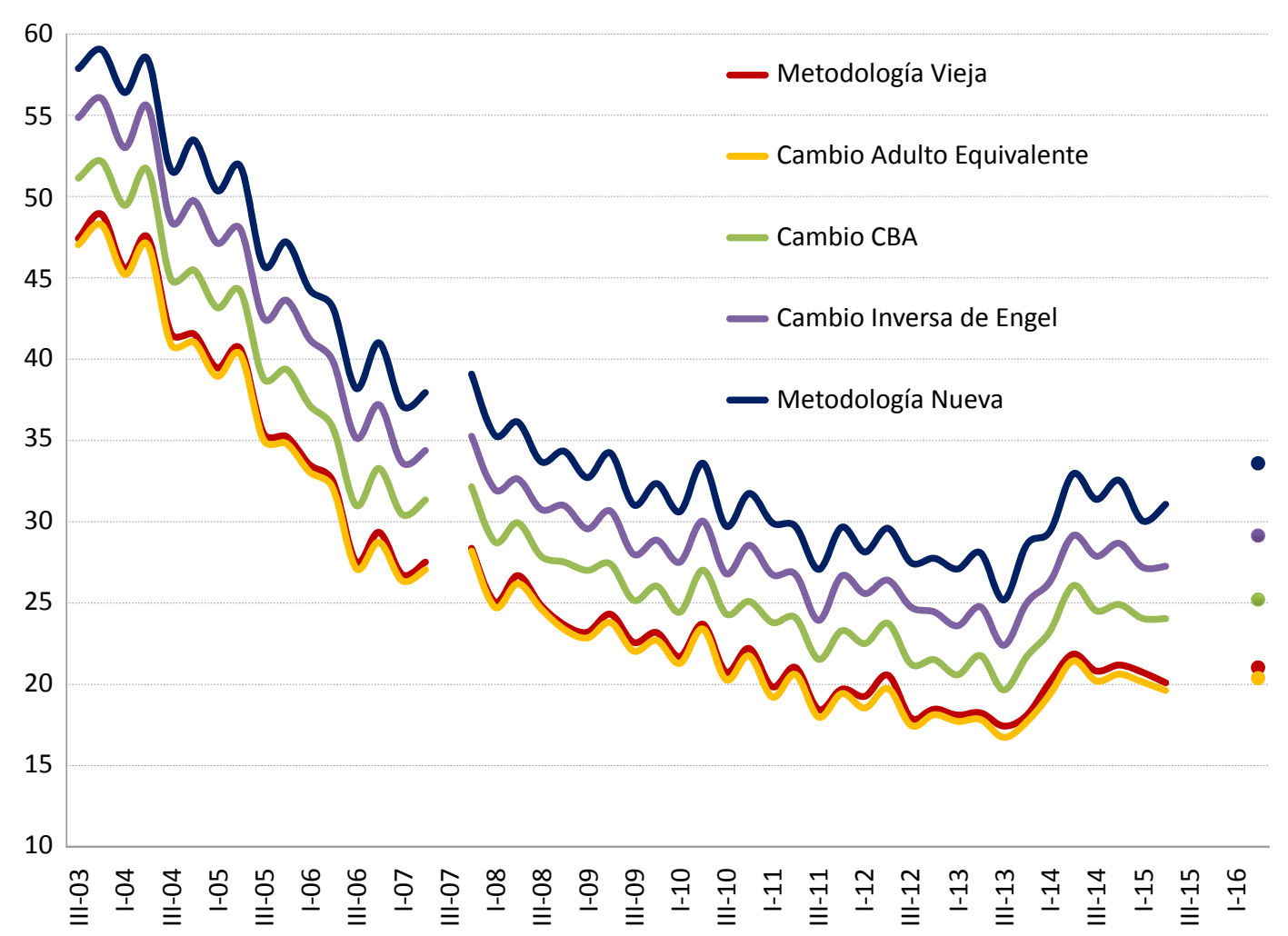

Fuente: elaboración propia con base a datos de EPH - INDEC

En el gráfico 2 se muestran las tasas de indigencia, cuya evolución es similar a las de pobreza, es decir, una caída fuerte desde valores elevados hasta 2007, luego una reducción a menor velocidad hasta 2012, desde cuando se observa un estancamiento y un aumento en 2014 y 2016. Nuevamente, el cambio de las necesidades energéticas en términos de adulto equivalente genera una modificación mínima hacia abajo en las tasas. Por su parte, la actualización de la CBA provo- 
ca un incremento de entre 2,6 y 1 p.p., con un promedio de 1,6 p.p. Asimismo, la suba en la ICE no impacta en la indigencia, sino únicamente en la pobreza, ya que solo afecta a la CBT dejando intacta a la CBA. De esta manera, el cambio total de la nueva metodología sobre las tasas de indigencia es apenas menor al registrado por la modificación en la CBA, ya que se le descuenta la nueva escala de las necesidades energéticas.

Gráfico 2: Tasa de indigencia (\% de la población total)

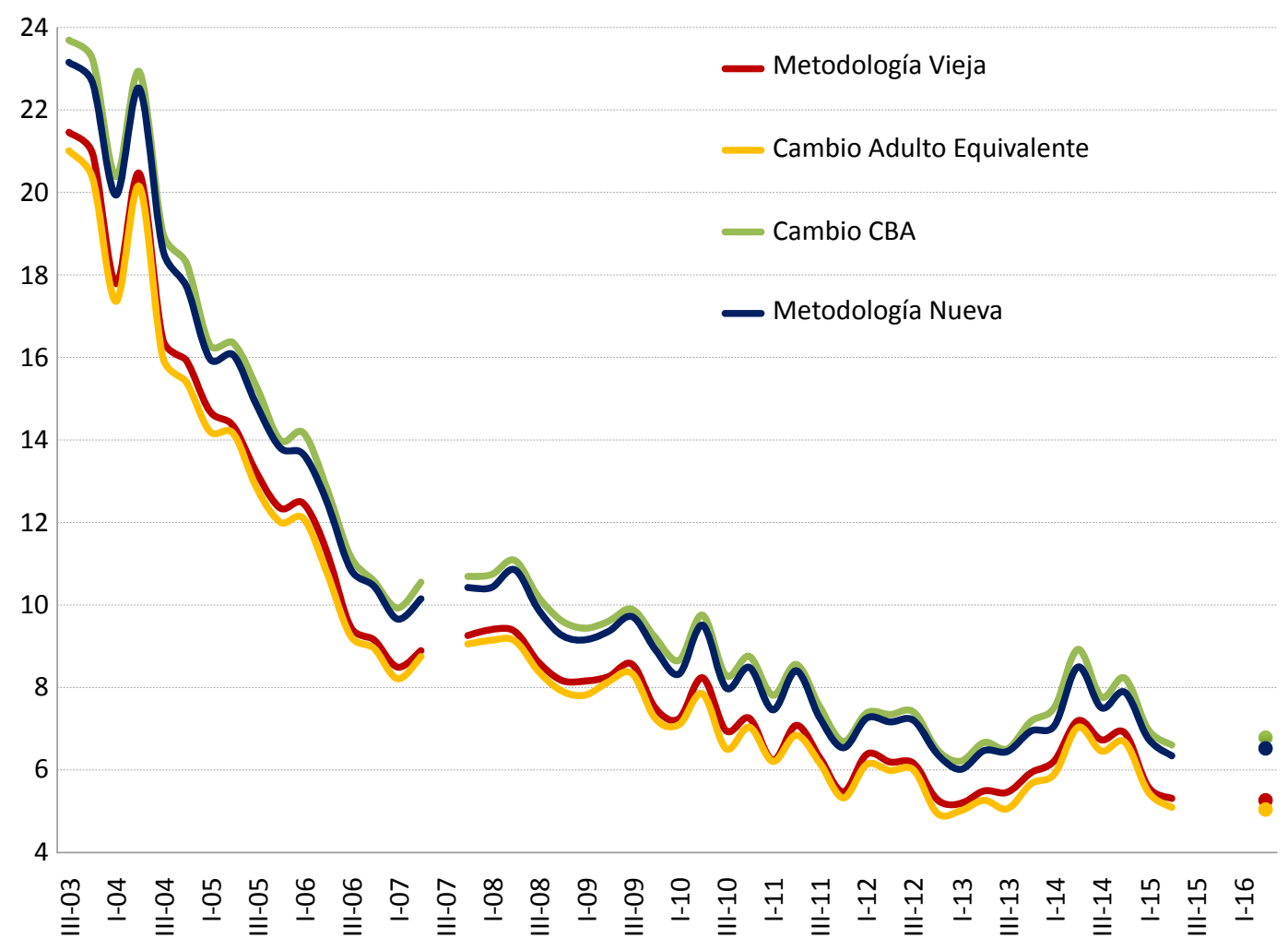

Fuente: elaboración propia con base a datos de EPH - INDEC

La brecha de pobreza (indigencia) mide el porcentaje mínimo del ingreso total de la Argentina necesario para eliminar la pobreza (indigencia). Como puede observarse en los gráficos 3 y 4, la evolución en el tiempo es nuevamente la misma: caída fuerte hasta 2007, desaceleración hasta 2012 y luego estancamiento con aumento en 2014 y 2016. En este caso es interesante destacar que mientras la incidencia de la pobreza y la indigencia es muy elevada, es decir, las tasas son altas por lo que son fenómenos que afectan a una parte importante de la población, la profundidad no es tan significativa. Con esto no se quiere afirmar que las condiciones sociales son buenas en la Argentina ni que tienen una fácil solución, sino simplemente que no es necesaria una parte tan elevada del ingreso total del país para sacar a todos los individuos de la pobreza y 
la indigencia. En el gráfico 3 se muestra que al segundo trimestre de 2016, según la metodología vieja, era necesaria una transferencia del $2,8 \%$ del ingreso total para eliminar la pobreza, mientras que este porcentaje se reduce a $0,43 \%$ para eliminar la indigencia. Como venía sucediendo, la modificación en la escala de necesidades energéticas no provoca grandes diferencias. Por su parte, la actualización en la CBA incrementa las anteriores cifras a 3,9\% y 0,57\%, respectivamente. El aumento en la ICE hace lo propio en forma más significativa en el caso de la pobreza a $5 \%$ del ingreso (como se vio previamente, en el caso de la indigencia no tiene relevancia). Finalmente, la metodología nueva considera que es necesario realizar una transferencia mínima de $0,55 \%$ y 6,7\% del ingreso total del país para eliminar la indigencia y la pobreza, respectivamente.

Gráfico 3: Brecha de pobreza (\% del ingreso total)

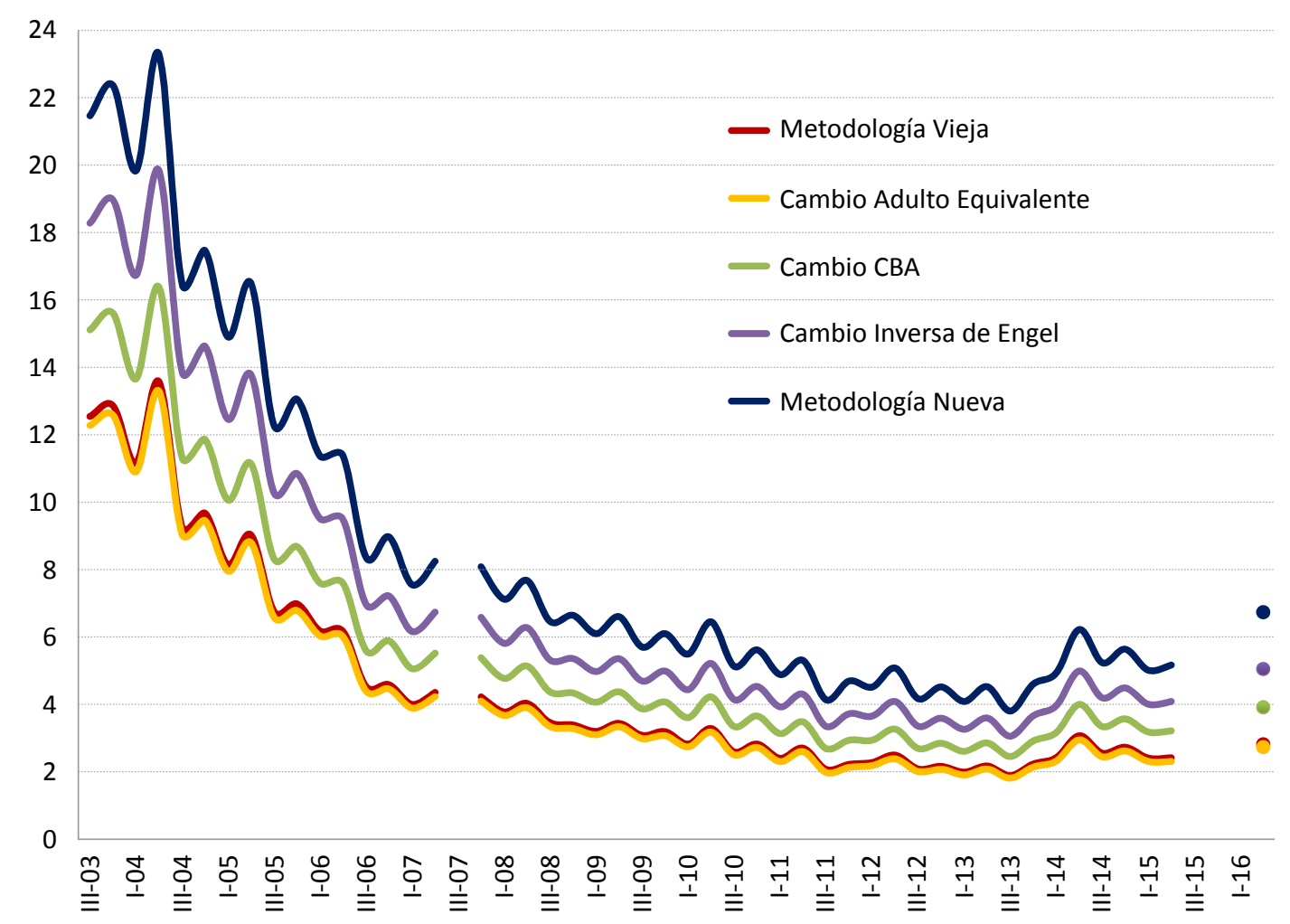

Fuente: elaboración propia con base a datos de EPH - INDEC

En definitiva, la actualización metodológica que dispuso el INDEC en las mediciones de pobreza e indigencia no alteraron la evolución de los indicadores, pero sí sus niveles. La modificación de la especificación de las necesidades energéticas en términos de adulto equivalente no parece haber generado grandes diferencias y, en cualquier caso, estas son a la baja. En cambio, la actualización de los patrones de consumo a partir del reemplazo de la ENGHo 1985/6 por la 2004/5 provocó un alza en los indicadores, tanto por la incorporación de nuevos productos 
en CBA y por la variación en las participaciones de sus componentes, como por el aumento en la ICE. Este último es sin lugar a dudas la actualización más relevante. Finalmente, no se pudo evaluar el impacto de la utilización de patrones de consumo específicos por región ante la falta de datos para calcular el valor de las respectivas CBA en los períodos pasados.

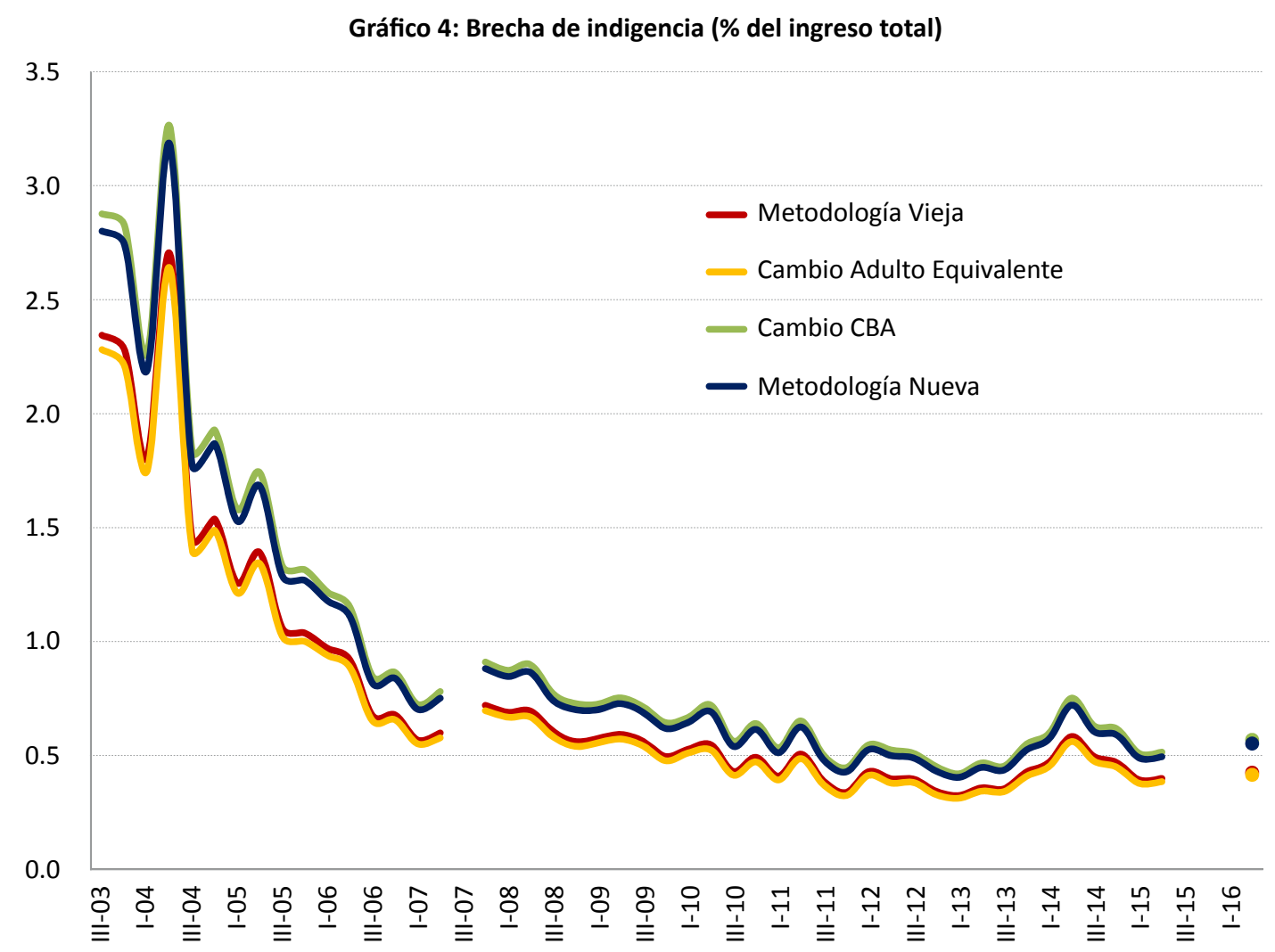

Fuente: elaboración propia con base a datos de EPH - INDEC

\section{POBREZA CERO, UN OBJETIVO CADA VEZ MÁS LEJANO}

Una de las promesas de campaña más mencionadas por el actual presidente de la Argentina, Mauricio Macri, fue la eliminación de la pobreza y, por lo tanto, también de la indigencia en el transcurso de sus cuatro años de mandato. Sus primeros meses no parecieron contribuir a dicho objetivo, pues - luego de la devaluación de diciembre - el nivel de actividad económica cayó y la inflación se aceleró, lo que tuvo un claro impacto sobre los ingresos. Es por eso que todos los indicadores de pobreza e indigencia vistos en el apartado anterior, tanto con la metodología nueva como con la vieja, presentan un deterioro en el segundo trimestre de 2016 en relación a un año atrás. 
Una economía tiene básicamente dos maneras de reducir la pobreza y la indigencia: creciendo y mejorando la distribución del ingreso. En el primer caso porque si el crecimiento se traduce en aumento de ingresos, habrá individuos cuyo ingreso supere las canastas básicas total y alimentaria y, por consiguiente, dejen de ser pobres e indigentes. En el segundo caso porque, aún sin crecimiento ni aumento de los ingresos promedio de la economía, si un individuo no pobre (indigente) transfiere parte de su ingreso a otro individuo pobre (indigente) y este gracias a ello supera la CBT (CBA), entonces la mejora en la distribución del ingreso habrá reducido la pobreza (indigencia).

Entonces, el objetivo de esta sección es calcular cuánto debería crecer y mejorar la distribución del ingreso de la Argentina para eliminar totalmente la pobreza y la indigencia, según la metodología vieja y la nueva. Para ello, se hará uso de las curvas de isopobreza e isoindigencia, que indican todas las combinaciones posibles de crecimiento y mejora en el coeficiente de Gini para que estos indicadores sociales se reduzcan en un determinado porcentaje, que en este caso es 100\% (eliminación total). Como al mandato de Macri le quedan básicamente tres años, se plantearán las combinaciones para lograr el objetivo en ese plazo. Pero como, tal como se verá seguidamente, el objetivo es muy ambicioso, también se evaluarán las posibilidades de conseguirlo en un plazo de siete años, es decir, al término de un eventual segundo mandato del actual presidente.

En el gráfico 5 se observa que, siguiendo la metodología vieja de medición de la pobreza, es necesario que la economía crezca y que la distribución del ingreso mejore a tasas gigantescas los próximos tres años para poder cumplir el objetivo de pobreza cero. A modo de ejemplo, si el coeficiente de Gini se reduce un 4\% por año en promedio, la economía debería crecer un promedio anual de más de 40\%. Si la posibilidad de incrementar los ingresos reales a razón del 40\% anual por tres años parece imposible, no lo es menos una caída del coeficiente de Gini del 4\%, ya que los indicadores de distribución del ingreso suelen evolucionar muy lentamente. Estos porcentajes aumentan aún más a partir de la nueva medición de la pobreza. En efecto, con los datos oficiales actuales y manteniendo el escenario de disminución del Gini de $4 \%$ por año, sería necesario que la economía crezca un promedio anual de más del 60\% de acá al fin del mandato presidencial.

En definitiva, es imposible que el actual gobierno pueda cumplir con la promesa de campaña. La economía argentina nunca presentó una mejora tan sustancial en tan poco tiempo, ni mucho menos tampoco. Es por eso que también en el gráfico 5 se muestra cuánto menores deberían ser estos porcentajes en caso de plantearse el objetivo en un plazo de siete años, en lugar de tres. Si bien el objetivo es bastante menor, aún sigue encontrándose muy por encima de cualquier experiencia pasada de la Argentina y de la mayor parte de los países del mundo. Según la metodología vieja, el país debería combinar una baja del Gini promedio del $2 \%$ con un crecimiento algo mayor al 10\% por año para eliminar la pobreza al cabo de 2023. Para dar una idea, estos guarismos no se lograron ni entre 2004 y 2011, cuando el país mostró el período de mayor crecimiento y mejora de la distribución del ingreso en décadas, partiendo de una situación mucho más alarmante y, en cierto punto, más fácilmente mejorable. Con la meto- 
dología nueva el objetivo es todavía más exigente. Manteniendo el escenario de reducción del coeficiente de Gini de $2 \%$ por año, los ingresos de la economía deberían crecer a un promedio del 20\% anual en los próximos siete años.

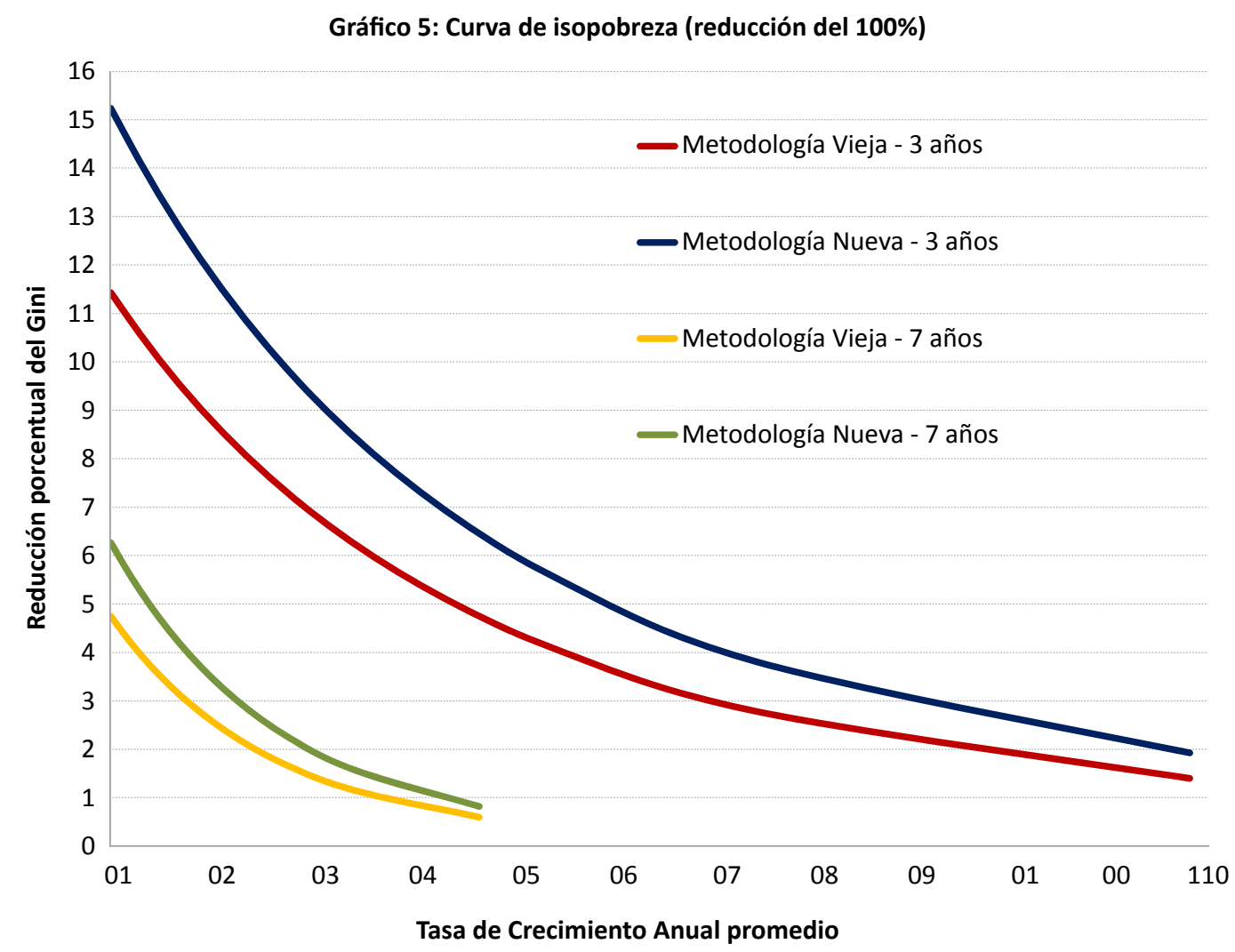

Fuente: elaboración propia con base a datos de EPH - INDEC

Si el objetivo se planteara sobre la indigencia en lugar de la pobreza, es decir, si fuera mucho menos exigente, aun así no parece probable que se pueda alcanzar. Como se observa en el gráfico 6, la curva de isoindigencia muestra que, bajo el escenario de una mejora del índice de Gini de $4 \%$ por año, la indigencia se vería erradicada hacia finales de 2019 solo en caso de que el crecimiento promedio anual sea mayor al $15 \%$, si se toma en consideración la metodología vieja, y mayor al $20 \%$, si se toma en consideración la metodología nueva. En caso de plantearse este mismo objetivo pero en un plazo de siete años, la exigencia disminuye, pero aun así se ve muy difícil de alcanzar. En efecto, solo con una mejora del Gini superior al 1,5\% anual, el crecimiento requerido sería menor al 10\% por año, tanto para la metodología vieja como para la nueva.

En definitiva, este sencillo análisis muestra que la promesa de campaña del actual presidente Mauricio Macri de terminar con la pobreza en la Argentina para el final de su mandato no es posible ser cumplida. Ni siquiera parece factible hacerlo sobre la indigencia y en un plazo de siete 
años. Para ello, la economía debería crecer a tasas exorbitantes y la distribución del ingreso debería mejorar a un ritmo nunca antes visto. En lugar de ello, el año pasado fue de caída del ingreso y, presumiblemente, deterioro de la distribución.

Gráfico 6: Curva de isoindigencia (reducción del 100\%)

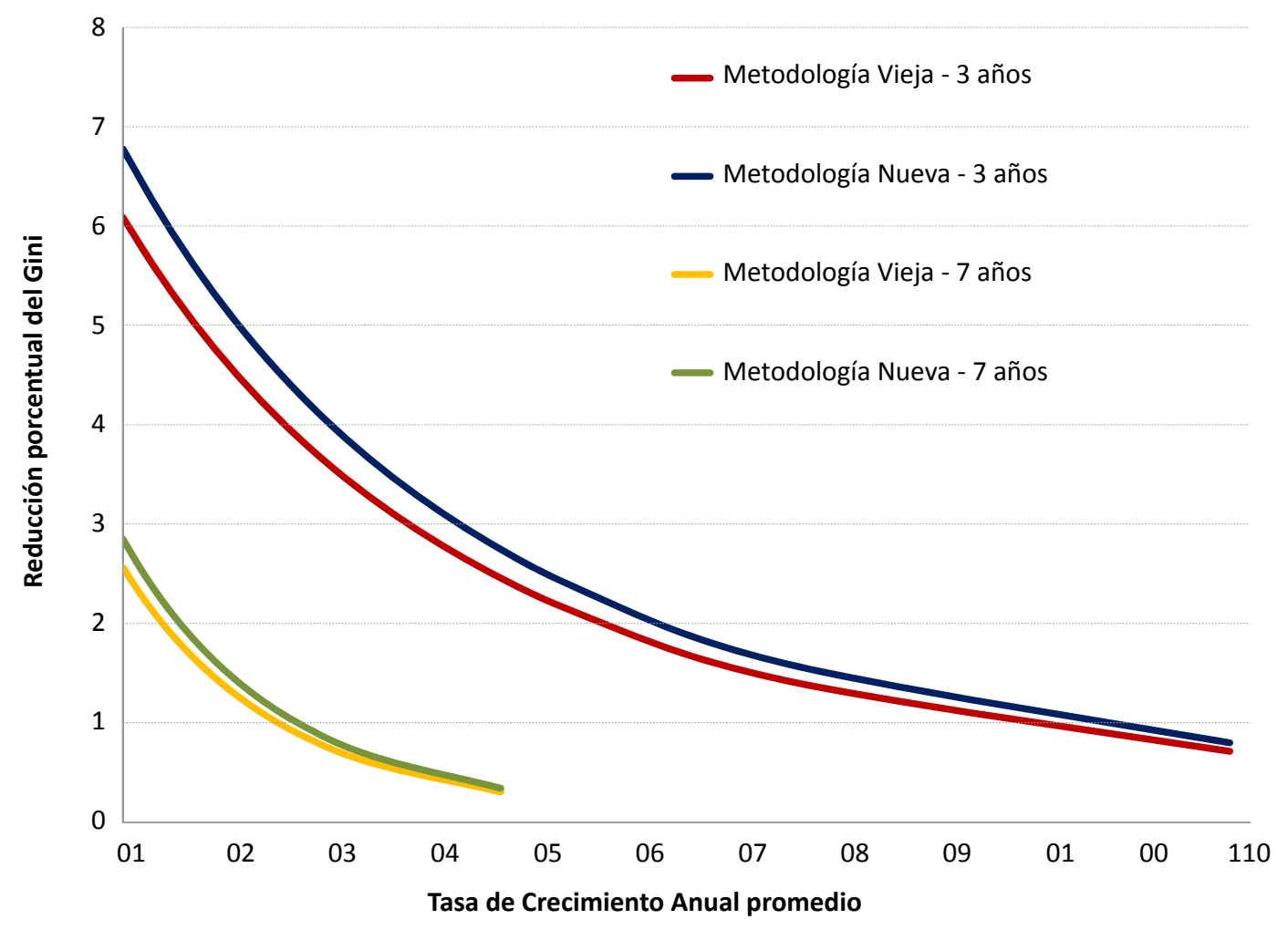

Fuente: elaboración propia con base a datos de EPH - INDEC

\section{REFLEXIONES FINALES}

El deterioro en la calidad de las estadísticas públicas de la Argentina se inició con la tasa de inflación, pero luego fue abarcando cada vez más indicadores que hacen uso de los niveles de precios como insumos. Uno de esos casos son los indicadores de pobreza e indigencia por ingresos, ya que estos últimos se comparan con las CBA y CBT, respectivamente, las cuales se actualizan con el relevamiento de precios del IPC. Así, la subestimación de la inflación llevó a una subestimación de las canastas y, finalmente, a mostrar una situación social mucho mejor a la real. En 2013, las series de tasas de indigencia y pobreza que publicaba periódicamente el INDEC se alejaban tanto de la realidad que se tomó la decisión de discontinuarlas. 
Luego del cambio de gobierno, en el marco de la normalización del instituto de estadística, se volvieron a publicar los indicadores sociales a partir del segundo trimestre de 2016. Pero, sorpresivamente, las tasas de indigencia y pobreza se situaron muy por encima de lo estimado por casi todas las consultoras privadas. El motivo de esta diferencia fue la aplicación de una actualización metodológica en el cálculo de los indicadores. Entre los cambios introducidos se destacan una mejor especificación de las necesidades energéticas en términos de adulto equivalente, la actualización de la CBA y de la ICE a partir del reemplazo de la ENGHo 1985/6 por la 2004/5, y la utilización de una CBA, ICE y CBT específica para cada región.

Todos estos cambios generan diferencias en el cálculo de los indicadores sociales. El único que tiene un efecto hacia la baja es la mejor especificación de las equivalencias energéticas, aunque su magnitud es muy reducida. Por su parte, la nueva CBA está integrada por nuevos productos y, los que se mantienen, en promedio aumentan su cantidad. De esta manera, la nueva línea de indigencia es más elevada y, por lo tanto, la indigencia y la pobreza es mayor. También se incrementó la ICE debido a que el gasto en alimentos y bebidas de la población de referencia representa una menor proporción del gasto total en comparación a la estructura de gasto anterior. Así, la CBT no solo ve aumentada por la mayor CBA, sino también por factor de expansión más alto. Como puede notarse, este último cambio es el más relevante en el caso de la pobreza, pero no tiene impacto sobre la indigencia. Por último, lamentablemente no se pudo evaluar el efecto de la especificación de los patrones de consumo por región ante la dificultad por encontrar datos regionales de precios que permitan calcular las canastas entre 2007 y 2015. No obstante, según el INDEC (2016b), este cambio habría generado una diferencia apenas mayor a 1 p.p. en la tasa de pobreza de 2006.

En definitiva, la actualización metodológica elevó la tasa de pobreza del segundo trimestre de 2016 en 12,6 p.p., mientras que hizo lo propio con la indigencia en 1,3 p.p. De esta manera, si ya con la metodología vieja la promesa de campaña del presidente Mauricio Macri de eliminar la pobreza para el final de su mandato parecía imposible, con la nueva metodología lo es aún más. Las curvas de isopobreza e isoindigencia calculadas en este artículo corroboran lo anterior, al mostrar que la mejora en la distribución del ingreso y el crecimiento que la economía argentina debería alcanzar para ello es de una magnitud inimaginable. De hecho, tampoco parece posible alcanzar un objetivo mucho menos ambicioso como eliminar la indigencia al cabo de siete años, menos todavía luego del año pasado en el que se observó una caída del ingreso y, presumiblemente, un dete-rioro en la distribución.

En conclusión, es una buena noticia que el INDEC vuelva a publicar datos de pobreza e indigencia, aunque el vacío estadístico oficial abarca un período demasiado grande (2007 - 2015) y eso va a provocar muchos inconvenientes tanto para evaluar el posible impacto de políticas de cara a la toma de decisiones, como para la actividad de la investigación. Más allá de eso, la actualización metodológica parece coherente y a priori no genera sospechas en relación a la calidad de los datos. En ese sentido, el deterioro en los indicadores sociales experimentado por el país en 2016, si bien evidente, es un tema alarmante que requiere de una atención urgente por parte del sector público. 
El punto principal es que la economía vuelva a crecer en forma sostenida, algo que no está para nada asegurado dado las características estructurales del país y el posible deterioro en el contexto internacional. Ante la falta de crecimiento, siempre se puede hacer uso de políticas sociales que mejoren la distribución del ingreso aunque, si bien la sociedad suele declarar estar a favor de ello, tiende a mostrar rechazo ante cualquier intento concreto.

\section{REFERENCIAS BIBLIOGRÁFICAS}

INDEC (2004). “Actualización de la metodología oficial de cálculo de las Líneas de Pobreza”. Documento en discusión presentado en el $13^{\circ}$ Taller Regional La construcción de líneas de pobreza en América Latina. Metodología y Práctica. Lima. http://www.cepal.org/deype/ mecovi/docs/taller13/4.pdf

INDEC (2016a). "Incidencia de la pobreza y de la indigencia en 31 aglomerados urbanos". http://www.indec.gov.ar/uploads/informesdeprensa/eph_pobreza_01_16.pdf

INDEC (2016b). “La medición de la pobreza y la indigencia en la Argentina”, Metodología INDEC, 22. http://www.indec.gob.ar/ftp/cuadros/sociedad/EPH_metodologia_22_pobreza.pdf

\section{CURRICULUM VITAE}

\section{Guido Zack}

Dr. en Análisis Económico Aplicado por las Universidades de Alcalá y Complutense de Madrid (España). Profesor e investigador del Instituto Interdisciplinario de Economía Política de Buenos Aires (UBA-CONICET) y de la Escuela de Economía y Negocios (UNSAM).

zack.gui@gmail.com

\section{Federico Favata}

Lic. en Economía por la Universidad de San Martín. Profesor e investigador de la Escuela de Economía y Negocios (UNSAM).

favata.federico@gmail.com

\section{Florencia Fares}

Lic. en Economía por la Universidad de San Martín. Profesora e investigadora de la Escuela de Economía y Negocios (UNSAM).

florenciamf9@yahoo.com.ar 
Tabla A.1: Composición de la Canasta Básica

Alimentaria para el adulto equivalente

\begin{tabular}{|c|c|c|c|}
\hline Productos & $\begin{array}{c}\text { Vieja } \\
\text { (gramos) }\end{array}$ & $\begin{array}{l}\text { Nueva } \\
\text { (gramos) }\end{array}$ & $\begin{array}{c}\text { Comparación Nueva } \\
\text { - Vieja (gramos) }\end{array}$ \\
\hline Pan & 6750 & 6060 & 690 \\
\hline Galletitas saladas & 420 & 420 & 0 \\
\hline Galletitas dulces & 210 & 720 & -510 \\
\hline Arroz & 1200 & 630 & 570 \\
\hline Harina de trigo & 1080 & 1020 & 60 \\
\hline Otras harinas (maíz) & 210 & 210 & 0 \\
\hline Fideos & 1740 & 1290 & 450 \\
\hline Papa & 6510 & 7050 & -540 \\
\hline Batata & 510 & 690 & -180 \\
\hline Azúcar & 1230 & 1440 & -210 \\
\hline Dulces (dulce de leche, dulce de batata, mermeladas) & 330 & 240 & 90 \\
\hline Legumbres (lentejas, porotos, arvejas) & 240 & 240 & 0 \\
\hline $\begin{array}{l}\text { Hortalizas (acelga, cebolla, lechuga, tomate, zanahoria, } \\
\text { zapallo, tomate en lata) }\end{array}$ & 5730 & 3930 & 1800 \\
\hline Frutas (banana, mandarina, manzana, naranja) & 4950 & 4020 & 930 \\
\hline Carnes (asado, carnaza, carne picada, espinazo, hígado, nalga, paleta, pollo) & 6270 & 6270 & 0 \\
\hline Huevos & 600 & 630 & -30 \\
\hline Leche & 9270 & 7950 & 1320 \\
\hline Queso (fresco, crema, cuartirolo, de rallar) & 330 & 270 & 60 \\
\hline Aceite (mezcla) & 1200 & 1200 & 0 \\
\hline Bebidas edulcorantes (jugos para diluir) & 4050 & 4050 & 0 \\
\hline Bebidas gaseosas sin edulcorar (soda) & 3450 & 3450 & 0 \\
\hline Sal fina & 120 & 150 & -30 \\
\hline Sal gruesa & & 90 & -90 \\
\hline Vinagre & 60 & 90 & -30 \\
\hline Café & 30 & 60 & -30 \\
\hline Té & & 60 & -60 \\
\hline Yerba & 510 & 600 & -90 \\
\hline \multicolumn{4}{|l|}{ Productos nuevos } \\
\hline Fiambres (paleta cocida, salame) & 60 & 0 & 60 \\
\hline Carnes (pescado) & 627 & 0 & 627 \\
\hline Yogur & 570 & 0 & 570 \\
\hline Manteca & 60 & 0 & 60 \\
\hline Bebidas alcohólicas (cerveza, vino) & 1080 & 0 & 1080 \\
\hline
\end{tabular}

Fuente: INDEC (2004) 
Tabla A.2: Necesidades energéticas y unidades consumidoras según edad y sexo

\begin{tabular}{|c|c|c|c|c|c|c|}
\hline & \multicolumn{3}{|l|}{ Nueva } & \multicolumn{3}{|c|}{ Vieja } \\
\hline Sexo & Edad & $\begin{array}{l}\text { Necesidad } \\
\text { Energética }\end{array}$ & $\begin{array}{c}\text { Unidades } \\
\text { consumidoras }\end{array}$ & Edad & $\begin{array}{l}\text { Necesidad } \\
\text { Energética }\end{array}$ & \begin{tabular}{|c|} 
Unidades \\
consumidoras
\end{tabular} \\
\hline \multirow{11}{*}{ Ambos } & 6-9 meses & 776 & 0.28 & Menor de un año & 880 & 0.33 \\
\hline & 9-12 meses & 952 & 0.35 & & & \\
\hline & 1 año & 1030 & 0.37 & 1 año & 1170 & 0.43 \\
\hline & 2 años & 1277 & 0.46 & 2 años & 1360 & 0.50 \\
\hline & 3 años & 1409 & 0.51 & 3 años & 1500 & 0.56 \\
\hline & 4 años & 1518 & 0.55 & 4-6 años & 1710 & 0.63 \\
\hline & 5 años & 1643 & 0.60 & & & \\
\hline & 6 años & 1760 & 0.64 & & & \\
\hline & 7 años & 1813 & 0.66 & 7- 9 años & 1950 & 0.72 \\
\hline & 8 años & 1865 & 0.68 & & & \\
\hline & 9 años & 1910 & 0.69 & & & \\
\hline \multirow{8}{*}{ Varones } & 10 años & 2182 & 0.79 & 10-12 años & 2230 & 0.83 \\
\hline & 11 años & 2266 & 0.82 & & & \\
\hline & 12 años & 2347 & 0.85 & & & \\
\hline & 13 años & 2472 & 0.90 & 13-15 años & 2580 & 0.96 \\
\hline & 14 años & 2650 & 0.96 & & & \\
\hline & 15 años & 2760 & 1.00 & & & \\
\hline & 16 años & 2828 & 1.03 & & & \\
\hline & 17 años & 2861 & 1.04 & 16-17 años & 2840 & 1.05 \\
\hline \multirow{8}{*}{ Mujeres } & 10 años & 1918 & 0.70 & 10-12 años & 1980 & 0.73 \\
\hline & 11 años & 1986 & 0.72 & & & \\
\hline & 12 años & 2061 & 0.75 & & & \\
\hline & 13 años & 2089 & 0.76 & 13-15 años & 2140 & 0.79 \\
\hline & 14 años & 2100 & 0.76 & & & \\
\hline & 15 años & 2116 & 0.77 & & & \\
\hline & 16 años & 2111 & 0.77 & & & \\
\hline & 17 años & 2124 & 0.77 & 16-17 años & 2140 & 0.79 \\
\hline \multirow{5}{*}{ Varones } & 18-29 años & 2826 & 1.03 & 18-29 años & 2860 & 1.06 \\
\hline & 30-45 años & 2758 & 1.00 & 30-59 años & 2700 & 1 \\
\hline & 46-60 años & 2760 & 1.00 & & & \\
\hline & $61-75$ años & 2288 & 0.83 & & & \\
\hline & más de 75 años & 2050 & 0.75 & más de 60 años & 2210 & 0.82 \\
\hline \multirow{5}{*}{ Mujeres } & 18-29 años & 2106 & 0.77 & 18-29 años & 2000 & 0.74 \\
\hline & 30-45 años & 2111 & 0.77 & 30-59 años & 2000 & 0.74 \\
\hline & 46-60 años & 2090 & 0.76 & & & \\
\hline & 61-75 años & 1860 & 0.68 & & & \\
\hline & más de 75 años & 1750 & 0.64 & más de 60 años & 1730 & 0.64 \\
\hline
\end{tabular}

Fuente: INDEC (2004) 